Rationale Indacaterol/glycopyrronium (IND/GLY, QVA149) is a combination of a long-acting beta- 2 agonist and a muscarinic receptor antagonist for the once-daily treatment of COPD. Here we assessed the effects of indacaterol/glycopyrronium on lung function and physical activity compared with placebo.

Methods We performed a randomised, two-period, cross-over study ( 21 days of treatment separated by a wash-out period of 14 days) with IND/GLY $110 \mu \mathrm{g} / 50 \mu \mathrm{g}$ or matching placebo. Lung function was measured by slow and forced spirometry. Physical activity was measured by an activity monitor (Bodymedia SenseWear Armband) over the last week of each treatment period. The primary endpoint was peak inspiratory capacity (IC) at the end of each treatment period (i.e., on Day 21). The coprimary endpoint was physical activity level as defined by daily activity-related energy expenditure ( $\mathrm{kcal} / \mathrm{day})$. Secondary endpoints included number of steps per day, duration of at least moderate activity per day, peak IC and $\mathrm{FEV}_{1}$ on Day 1 , trough IC on Day 1, and trough IC and $\mathrm{FEV}_{1}$ on Day 21.

Results 194 patients (mean age 63 years; mean postbronchodilator $\mathrm{FEV}_{1} 61.6 \%$ predicted), were randomised; 183 patients completed the study. Peak IC on Day 21 was 0.202 L greater with IND/GLY compared to placebo ( $p<0.001$; Table 1$)$. In addition, superiority of indacaterol/glycopyrronium over placebo with regard to other parameters of lung function was demonstrated (Table 1). Compared with placebo, indacaterol/glycopyrronium significantly increased the change from baseline in average physical activity level with a difference of $36.7 \mathrm{kcal} /$ day. Further, IND/GLY -treated patients completed significantly more steps per day with a difference between the two treatment groups of 358.0 steps per day (Table 1 ).

Conclusion Compared with placebo, IND/GLY improved lung function and physical activity in patients with moderate to severe COPD.

\begin{tabular}{|c|c|c|}
\hline & & IND/GLY vs PBO \\
\hline \multirow[t]{4}{*}{ IC (Day 21) } & peak (primary) & $0.202 \mathrm{~L}$ \\
\hline & & $\mathrm{p}<0.001 ; 95 \% \mathrm{Cl}: 0.158-0.246$ \\
\hline & trough & $0.198 \mathrm{~L}$ \\
\hline & & $\mathrm{p}<0.001 ; 95 \%$ Cl: $0.151-0.245$ \\
\hline \multirow[t]{2}{*}{ IC (Day 1) } & peak & $0.260 \mathrm{~L}$ \\
\hline & & $\mathrm{p}<0.001 ; 95 \%$ Cl: $0.219-0.297$ \\
\hline \multirow[t]{2}{*}{$\mathrm{FEV}_{1}$ (Day 1) } & peak & $0.220 \mathrm{~L}$ \\
\hline & & $\mathrm{p}<0.001 ; 95 \%$ Cl: $0.189-0.251$ \\
\hline \multirow[t]{4}{*}{$\mathrm{FEV}_{1}$ (Day 21) } & peak & $0.136 \mathrm{~L}$ \\
\hline & & $\mathrm{p}<0.001 ; 95 \% \mathrm{Cl}: 0.102-0.170$ \\
\hline & trough & $0.277 \mathrm{~L}$ \\
\hline & & $\mathrm{p}<0.001 ; 95 \%$ Cl: $0.244-0.311$ \\
\hline \multirow{2}{*}{\multicolumn{2}{|c|}{$\begin{array}{l}\text { Activity related energy expenditure (kcal/day) } \\
\text { (co-primary) }\end{array}$}} & 36.713 \\
\hline & & $\mathrm{p}=0.0399 ; 95 \% \mathrm{Cl}: 1.724-71.701$ \\
\hline \multirow{2}{*}{\multicolumn{2}{|c|}{ Average physical activity level (PAL) }} & 0.0237 \\
\hline & & $\mathrm{p}=0.0191 ; 95 \%$ Cl: $0.004-0.043$ \\
\hline \multirow{2}{*}{\multicolumn{2}{|c|}{$\begin{array}{l}\text { Change from baseline in average number of steps } \\
\text { (Friedman's test) }\end{array}$}} & 358.0 \\
\hline & & $p=0.0288 ;(S D=2457.95)$ \\
\hline \multirow{2}{*}{\multicolumn{2}{|c|}{$\begin{array}{l}\text { Duration of at least moderate activity per day } \\
\text { (min/day) }\end{array}$}} & 4.382 \\
\hline & & $\mathrm{p}=0.2637 ; 95 \% \mathrm{Cl}:-3.333-12.098$ \\
\hline
\end{tabular}

\section{P141 AN EVALUATION OF THE ACCEPTABILITY OF SUPERVISED WARD-BASED EXERCISE FOR PATIENTS ADMITTED TO HOSPITAL FOR ACUTE EXACERBATION OF COPD}

L Hogg, S Madden-Scott, J Turnbull, L Osman. Guy's and St Thomas' NHS Foundation Trust, London, UK

\subsection{6/thoraxjnl-2015-207770.278}

Introduction Patients with COPD demonstrate peripheral muscle dysfunction and reduced physical activity. Both are compounded by admission for acute exacerbation (AECOPD). Supervised exercise during AECOPD has been shown to be safe and may ameliorate these deleterious physical effects. Debate remains as to the acceptability of exercise for patients admitted with AECOPD.

Objective To evaluate the acceptability of supervised exercise for patients admitted with AECOPD.

Methods Patients admitted with AECOPD between December 2013 and August 2014 were included if medically stable, had no other limiting factor to exercise and consented to participate. Physiotherapists prescribed a standardised progressive exercise programme comprising daily upper/lower limb strengthening exercises and walking, supervised by a physiotherapy assistant. Patients completed a self-reported Likert scale questionnaire on discharge. Data collection included MRC Dyspnoea score, COPD Assessment Test (CAT), Timed Up and Go (TUAG) and 4-metre gait speed (4MGS).

Results 150 patients were screened, 78 (52\%) participated. Mean (SD) age 70(10) years, 50\% female, median (IQR) length of stay 7(5 -12) days, median number of exercise sessions 2(13). Median MRC 4(4-5) ( $\mathrm{n}=60)$; mean CAT at baseline 26 with a mean change of $-3.7(n=50)$.

71 patients completed the questionnaire. $89 \%$ felt happy to participate in exercise when approached by a physiotherapist. 93\% reported being able to undertake the exercises taught, $80 \%$ felt very or fairly confident to continue at home. $82 \%$ felt the exercise improved their ability to carry out functional tasks. 34\% recalled previously completing Pulmonary Rehabilitation.

Analysis of those who completed TUAG and 4MGS pre and post intervention $(\mathrm{n}=15)$ showed mean baseline values of 23.7 (10.7) secs and $0.44(0.21)$ mps respectively; mean changes of 6.8(9.45) secs and $+0.08(0.16)$ mps respectively.

Conclusions Supervised exercise is acceptable to patients admitted with AECOPD, even in those demonstrating significant frailty. However, the non-participation rate was high, reasons for which are unknown. It is unclear whether the improvement in health status and functional mobility during admission was due to exercise participation or natural recovery. Further work is required exploring the impact of initiating exercise during admission on physical activity behaviours post discharge as well as reasons for non-participation during admission.

\section{P142 REDUCED ALL CAUSE HEALTHCARE UTILISATION AFTER BREATHING RETRAINING FOR DYSFUNCTIONAL BREATHING}

FC Thomson, DJ Ford. Scarborough General Hospital, York Teaching Hospital NHS Foundation Trust, UK

10.1136/thoraxjnl-2015-207770.279

Introduction There are few controlled studies to prove the effectiveness of breathing retraining in the management of 